\title{
The Physical Fitness Development of Children with Functionally Single Heart Ventricle as a Basis of the Physical Rehabilitation Technology After a Hemodynamic Correction
}

\author{
Dao Chanh Thuc ${ }^{1 *}$, Hang Quang Thai ${ }^{2}$ and Doan Tien Trung ${ }^{3}$ \\ 1,2Deparment of Physical Education, An Giang University, Vietnam \\ ${ }^{3}$ Deparment of Physical Education, Ho Chi Minh City University of Education, Vietnam
}

Received: 眥 November 27, 2018 Published: 海 December 03, 2018

*Corresponding author: Dao Chanh Thuc, Department of Physical Education, An GIANG University, Vietnam

\begin{abstract}
The aim of the performed research was to determine the features of the physical development and its connection with the body of the children of school age who have a congenital heart disease with a functionally single heart' ventricle for developing the physical rehabilitation technology. There were performed an examination of the 179 children with functionally single heart' ventricle hospitalized for operative treatment in the State Center «Scientific and Practical Medical Center of the Pediatric Cardiology and Cardiosurgery of the Ministry of Health of Vietnam in the 2016 and 2017 years. The analysis of the physical development indicators of the children with functionally single heart' ventricle allowed to establish a more pronounced lagging in the physical development by dividing the length and body mass index into the centile corridors and Roerre's index, comparing with children with other congenital heart disease, determining the need for development for patients with functionally single heart' ventricle special physical rehabilitation technology. The physical rehabilitation technology of the children with functionally single heart' ventricle after a hemodynamic correction should include the principles of physical rehabilitation, pedagogical principles, age characteristics of children, the indicators of physical development, the quantitative indicators of the biogeometrical posture profile, features of hemodynamics, functional condition of cardiovascular and respiratory systems.
\end{abstract}

Keywords: Physical Rehabilitation; Congenital Heart Disease; Functional Single Ventricle

\section{Introduction}

The valuation of the physical rehabilitation (PR) in the solving the tasks of restoring the functional condition of the body's systems and patients' health after the surgical intervention of the congenital heart disease (CHD), including such anomaly as a functionally single heart' ventricle (FSHV) [1]. The literature contains a lot of the publications describing the surgical tactics of the correction phase of the hemodynamics during FSHV, but the case about the PR process of such children before and after these operations are not adequately reflected [1].

Rhodes [2] and Swan [3] recommended the restriction of motor activity after surgical intervention at the early and removal stages of recovering process, the case about the using of the physical tools of a rehabilitation is ignored, and recommended rehabilitation programs have no complex character, focusing on a certain stage of rehab and/or one of the parts of physical health directly related to the pathology, described in the case [1]. Bringing that integrated programs based on a preliminary analysis of physical development, posture profile and physiological systems have a low level of implementation in specialized medical and rehabilitation institutions, have proved their effectiveness in the physical rehabilitation of children with CHD and the importance of the effective restoration of the body of children with FSHV after performing hemodynamic correction creates the need to develop a rehabilitation technology for the using in the physical rehabilitation process this contingent of patients.

The presence in the people with FSHV the lagging in physical fitness and related reducing the functionality of the respiratory system [4], the low tolerance to physical activity [5] and the disorders of the psychosomatic development and reduction of social activity [6] make these features system-forming for the formation of the physical rehabilitation technology of children with FSHV after a hemodynamic correction. 


\section{Purpose of the Research}

To determine the features of the physical development and its connection with the body of the children of school age who have a CHD with a FSHV for developing the physical rehabilitation technology.

\section{Materials and Methods}

The determination of the physical development features was done with using the centimeters of body length and body weight, Roerre's index (RI) of the 179 children with FSHV hospitalized for operative treatment in State Center «Scientific and Practical Medical Center of the Pediatric Cardiology and Cardiosurgery of the Ministry of Health of Vietnam in the 2016 and 2017 years. We used a six-minute walking test (6MWT) with the registration of the walked distance, registration of the heart rate (HR) and the indicator of the blood saturation index (Sp02) were used to determine the indicators of the functional condition of the cardiac and vascular system in children with FSHV. For determining the functional condition of the respiratory system, a spirograph was used as the Spirolab III (Italy) with the automatic determination of indicators in percentage from the norm [1]. There were recorded indicators of life capacity of the lungs (VC), capacity of the inhalation (VTin) and exhalation (VTex), the reserve volumes of inhalation (RVTin) and exhalation (RVTex), the flow-volume curve indicators (FEV1, FEF25, PEFin), with the subsequent calculation of the Tifno index (FEV1,/FVC) [1].

For determining of the disorders in the posture, we have analyzed the parameters of the biogeometrical profile in the frontal and sagittal planes. The assessment of the quality of life in the physical functioning area (PF) and psychosocial functioning (PSF) was performed with the using an international standardized questionnaire PedsQLTM 4.0 Generic Core Scales (France) either in children or in parents.

\section{Results}

The analysis of the physical development indicators of the children with FSHV allowed establishing a more pronounced lagging in the physical development by dividing the length and body mass index into the centile corridors and RI, compared with children with other CHD, determining the need for development for patients with FSHV special physical rehabilitation technology. The distribution by the RI in the children with FSHV followed: low level indicates on body thinness in the $31.05 \%$; the medium level indicates on the harmony between the mass ratio and body length in the 57.14\%; high level indicates on the excessive body weight in the $11.54 \%$.

In the detailed analysis of the specifics of the clinical condition, the functional condition of the cardiac and vascular system and respiratory system, the biogeometric profile of the posture and quality of life in children with FSHV, we can state the general condition of children at the time of admission to the clinic was assessed by the physician as satisfactory at $50.93 \%$, the moderate severity condition was in $47.96 \%$. The analysis of medical cards revealed that dyspnoea was observed in the $81.88 \%$ of children, acrocyanosis was in the $40 \%$, generalized cyanosis was in the $34.34 \%$, and cyanosis was observed in the $25.67 \%$. Me indicator (25\%; 75\%) of the hemoglobin was higher than normal and made

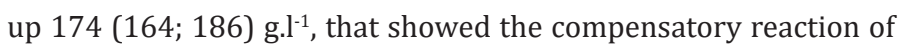
the body on the mixing of venous and arterial blood in children with FSHV. According to echocardiographic studies, good ventricular contractility was observed in the 32 (as the 91.44\%) children, and satisfactory only in the 3 (as the 8.56\%).

The result obtained from the analysis of the length of the walked distance during the 6MWT performance showed, that the results do not correspond to the age and sex norms. Children with FSHV per six minutes walked $461.89 \pm 53.98 \mathrm{~m}$. The adequate increasing of the HR indicator (20-40\%) after the 6MWT in children of the studied group was recorded in $40 \%$ of cases, the insufficient increasing $(<20 \%)$ was in the $20 \%$, and excessive increasing $(>40 \%)$ was in the $40 \% . \mathrm{SpO}_{2}$ indicator before the $6 \mathrm{MWT}$ was $78.92 \pm 6.79 \%$, as the result of the mixing of the venous and arterial blood. After the performing, the 6MWT were fixed $(\mathrm{p}<0.001)$ decreasing the SpO2 indicator on the $9.07 \pm 3.95 \%$, indicating on the instability and sensitivity of this indicator to physical activity.

The indicators characterizing the functional condition of the respiratory system reduced in children with FSHV. VC indicator was on the $75.93 \pm 10.75 \%$ from the proper level. In accordance with the clinical rules, $17.13 \%$ of the children had a normal indicator of the VC, $28.56 \%$ had a conditionally normal same indicator, $40 \% \mathrm{~h}$ a d moderately reduced the same indicator, $14.31 \%$ had significantly lowered same indicator. VTin indicator in the relative terms was $84.81 \pm 19.77 \%$, RVTex indicator was $63.66 \pm 23.69 \%$, the volume of forced exhalation for the first second (FEV1) was $77.01 \pm 12.91 \%$. The analysis of the data of the Tifno index get indicator of the 89.74 $(81.19 ; 93.02) \%$ it was as close as possible to the norm. The relative value of peak volume exhaust velocity was $72.48 \pm 18.97 \%$, which is a conditional norm. Indicator of the FEF25, reflecting the passage of the respiratory tract at the level of large bronchi and trachea was the $68.41 \pm 18.12 \%$ from the proper value. The injected vital capacity of the inhalation was $65.04(59.02 ; 79.02) \%$ from the proper value, the volume of forced inhalation in the first second was $68.12 \pm 13.87 \%$, and the PEFin indicator was $45.76 \pm 11.72 \%$. Individuals had disorders in the frontal plane, $97.1 \%$ of individuals had disorders in the sagittal plane. In the frontal plane, the must increase value had such angles: $\beta 2$ angle $\left(3.10 \pm 1.58^{\circ}\right)$, characterizing the symmetry of the shoulder gird and made by the line of the horizon and the line between the acromonies, and $\beta 3$ angle $\left(4.63 \pm 2.29^{\circ}\right)$, made by the line of the horizon and the line connecting the points of the lower corners of the shoulder blades. The statistical indicator 
Me (25\%; 75\%) was $2.77(2.00 ; 4.23)^{\circ}$ for $\beta 2$ angle and 4.30 $(3.02 ; 5.95)^{\circ}$ for $\beta 3$ angle. All angles characterizing posture in the sagittal plane have also been increased. The $\alpha 2$ angle value, made by the intersection of the vertical line and the line between the acromion and the infra-red point, that characterizing the thoracic spine, was $8.24 \pm 4.29^{\circ}$; Me $(25 \% ; 75 \%)=7.95(5.12 ; 11.51)^{\circ}$, that confirming hyperkyphosis. The $\alpha 4$ angle value made by a vertical line intersection and a line between the center of the crest of the iliac bone and the trochanterion, was $7.36 \pm 4.49^{\circ} ; \mathrm{Me}(25 \% ; 75 \%)=$ $7.31(3.79 ; 11.05)^{\circ}$, that confirmed lower hyperlordosis.

In the physical functioning area (PF), the assessment of the quality of life, according to the child form of the questionnaire, established low values of indicators, it was $54.89 \pm 10.91$ points, while other indicators were significantly lowered $(p<0.01)$. The value of the scale of psychosocial functioning (PSF), generally reflecting the emotional, social and role function was $69.24 \pm 10.56$ points. Total Quality of Life Score was $64.57 \pm 9.25$ points.

The quality of life of the examined children was not significantly different from the estimates of the child and their parents ( $p>0.05$ ). Particularly, during the studying of the quality of life of examinated children, according to the data from the parent form of the questionnaire, it was discovered that the PF value is $53.05 \pm 16.69$ points, and the total score was $63.12 \pm 12.52$ points.

\section{Discussion}

Results of the performed research confirm data, obtained from $[7,8]$ about the widespread lagging in the physical development among children with CHD as a FSHV. For such contingent of patients, there is a typical decrease in the tolerance to physical activity, the prevalence of posture disorders, and the relatively reduced functionality of the RS, that's not restored to the initial values at the time of discharge from the clinic [1,9].

The obtained data about features of the distribution of indicators of length and weight of a body on the centile corridors and RI indicator among children with FSHV became the basis for the scientific substantiation of the physical rehabilitation technology for children with such heart defects [1]. Such a technology should be made and realized in two phases (as the clinical and post-clinical), consists of the basic and variative components, depending on the individual characteristics of the physical development of children. The basic component is presented in the form of procedures of the therapeutic exercises, medical dosed walking, inhalations with a combined with usinth the physical rehabilitation tools, followed component should be aimed at early activation of patients after surgery, accelerate postoperative improvement of the functional condition of the cardiovascular system and RS [1].

The variative component should be aimed at the formation of the correct posture and the providing for the using of the physical rehabilitation tools, bringing the individual characteristics of the biogeometric profile of the patient's posture, and should include physical exercises and positioning, followed on the correcting of the pathological bends of the spine and consolidating the skills of correct posture $[1,3,10]$. The composition of the variable component of the physical rehabilitation technology should be formed in accordance with the fixed disorders of posture in the sagittal and frontal planes.

\section{Conclusion}

The well-known importance of the physical rehabilitation process in solving the problems of restoring the functional condition of the body's systems and patients' health after the surgical treatment of CHD, including FSHV, it creates the need for developing a physical rehabilitation technology for this contingent of patients, bringing their physical development [1]. In the most cases, rehab programs are not comprehensive, they are focusing on a certain stage of the rehab process and on one side of physical health, as the directly related to the pathology in the case.

The complex programs built on a preliminary analysis of the physical development, posture, and physiological systems, they have a low level of implementation in the specialized medical and rehabilitation institutions. During the admission to the clinic for the hemodynamic correction of the children with the FSHV, the following features of physical development are recorded, there are followed: the lagging in the physical development by the distribution of body length and body mass indexes on the centile corridors and according to RI, from the age norms. We recorded decreasing in the length of the walked distance during the 6MWT, with an adequate increasing of the HR after the physical activity in the $40 \%$ of cases, insufficient increasing was in the $20 \%$, excessive increasing was in $40 \%$ of children. Also, there was recorded decreased the functional capabilities of the respiratory system. An important feature of physical development is the presence of posture disorders in the frontal and sagittal planes in the children with FSHV. We marked changes in the biogeometric profile of posture in the frontal plane in the symmetry of the shoulder girdle and in the symmetry of the angles of the shoulder blades. In the sagittal plane, thoracic hyperkyphosis was recorded in all children. The deterioration of the quality of life, according to the PedsQLTM 4.0 Generic Core Scales questionnaire was the greatest difference in the physical functioning area both according to the data of children's and parental forms of questionnaires [1]. During the study, there was no statistically significant difference between the indicators according to the data of children's and parents' forms of questionnaires $(p>0,05)$.

\section{References}

1. Volodymyr Vitomskiy, Volodymyr Kormiltsev, Iryna Hruzevych, Svitlana Salnykova , Yurii Shevchuk, et al. (2018) Features of the physical development of children with functionally single heart ventricle as a basis of the physical rehabilitation technology after a hemodynamic correction. Journal of Physical Education and Sport 18(1): 421-424. 
2. Rhodes J, Tikkanen AU, Jenkins KJ (2000) Exercise Testing and Training in Children with Congenital Heart Disease. Circulation 122(19): 19571967.

3. Swan L, Hillis WS (2000) Exercise prescription in adults with congenital heart disease: A long way to go. Heart 83(6): 685-687.

4. Kul'chenko IA, Komisarenko OA (2012) Features of rehabilitation of patients who underwent coronary shunting. Cardiosurgery and inertial cardiology 1: 50-52.

5. Lazareva E, Vitomskiy V (2014) Means of physical rehabilitation at the inpatient stage of restoring the health of children operated on for congenital heart disease: The young scientific scholar of East-European National University named after L. Ukrainki. Series: physical education and sport 13: 37-42.

6. Drozdova IV, Lyisunets EM, Moroz SM (2010) Personal features of patients with operated congenital heart disease. International medical journal 16(4): 8-10.

7. Struchkova A (1975) Pediatric Thoracic Surgery. Medicine, pp .560.

8. Chaykovska GS, Gnateyko OZ (2012) Analysis of mortality and increased factors the risk of birth of children with congenital heart disease. Childss health 4: 67-70.

9. Larsson ES, Eriksson BO, Sixt R (2003) Decreased lung function and exercise capacity in Fontan patients. A long-term follow-up. Scandinavian Cardiovascular Journal 37(1): 58-63.

10. Vitomskiy V (2015) Methodical bases for constructing a program for physical rehabilitation for school-age children with a functionally single ventricle of the heart, the young scientific scholar of East-European National University named after. L. Ukrainky. Series: physical education and sport, 18: 111-116.

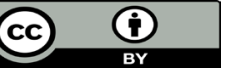

This work is licensed under Creative Commons Attribution 4.0 License

To Submit Your Article Click Here:

Submit Article

DOI: $10.32474 /$ OSMOAJ.2018.02.000135

$\begin{gathered}\text { Orthopedics and Sports Medicine } \\ \text { Open Access Journal }\end{gathered}$
Assets of Publishing with us
- Global archiving of articles
- Immediate, unrestricted online access
- Rigorous Peer Review Process
- Authors Retain Copyrights
- Unique DoI for all articles

